\title{
König Ludwig I. Urkunde von 1380
}

\author{
über das Asylrecht der \\ Marienburger Kirche. \\ Von
}

\section{Franz Zimmermann.}

$\mathrm{Zu}$ den ältesten urkundlich genannten Gemeinden der Umgebung Kronstadts, des Burzenlandes (terra Barcza, districtus Brassoniensis), gehört das auf dem äussersten Ausläufer des längs dem Homorodbache gegen den Altfluss zu sich erstreckenden Hügellandes gelegene Marienburg. Nur einige Jahrzehnte später, nachdem Deutsche ihren Fuss in den öden Südosten Siebenbürgens gesetzt und abendländische Cultur dahin gebracht hatten (1211)1), wird Marienburg als castram sanctae Mariae erwähnt. König Bela IV. verleiht nämlich im Jahre 1240 dem Cistercienserorden das Patronatsrecht über die Kirchen zu Marienburg, Petersberg, Honigberg und Tartlau ${ }^{2}$ ). Der Ort, bald als villa, bald als oppidum bezeichnet, kommt in zahlreichen Ürkunden vor, von welchen nicht wenige die Hattertgrenzen gegen die Nachbargemeinden Rothbach und Helsdorf betreffen. Es währte geraume Zeit, bis die Ansprüche der an den Grundstreitigkeiten betheiligten Gemeinden zur Ruhe kamen und die Stammesgenossen des häuslichen Haders müde sich vereinigten zu tapferer $\mathrm{Abwehr}$ des Alle gleich bedrohenden Halbmondes. Im Jahre 1371 erscheint der Ort als Mêren-

1) Teutsch und Firnhaber, Siebenbürg. Urkb. I. 8 f. - F. K. Schuller, Die deutschen Ritter im Burzenlande in Schuller, Archiv I. 161 f. - Philippi, Die deutschen Ritter im Burzenlande. Kronstadt 1861. \%) Teutsch und Firnhaber a. a. O. I. 68 nach Fejér Codex diplomaticus IV. 3. 550. Das von Letzterem als in Zircz befindlich bezeichnete Original dieser Urkunde befindet sich nach gefälliger Mittheilung des Herrn Abtei-Secretärs Mészáros nicht dort, und anch die daselbst verwahrten Abschriften von Urkunden betreffen nicht siebenbürgische Kirchen. 
burch $^{1}$ ), 1377 als Mergenburg $\left.{ }^{2}\right), 1378$ als Mergenborg $\left.{ }^{3}\right), 1413$ als Merenburch ${ }^{1}$ ), 1455 als Mergenburg ${ }^{4}$ ), in den königlichen Urkundeı des 14., 15. und 16. Jahrh. in maggarischer Benennung als Feldwar. oder Fewldwar ${ }^{5}$ ). Während dasselbe noch im 14. Jahrh. in Hermannstadt Recht sucht, einmal auch im 15. Jahrh., gehört Marienburg sei† 1377 sicher der politischen Gaugemeinschaft des Burzenlandes an, indem König Ludwig I. im Jahre 1377 alle dreizehn deutschen Gemeinden um Kronstadt als zu dieser Stadt gehörig, eidem civitati annexae, erklärt und auch die betreffende Urkunde an die seniores, iudices, iurati totaque communitas Saxonum sedis Brassouiensis richtet6).

Marienburg nimmt schon früh eine bedeutende Stelle unter den Nachbargemeinden ein. 1379 wird ihm von König Ludwig I. das Recht, an jedem Donnerstag Wochenmarkt abzuhalten ${ }^{7}$ ), und Ludwigs Nachfolger bestätigen dieses Recht. Der Arm des Marienburger Richters reichte über das Gemeindegebiet hinaus, denn Processe aus Helsdorf, Rothbach und Nussbach gingen im Falle der Berufung zunächst nach Marienburg ${ }^{8}$, erst in weiterer Instanz nach Kronstadt an den Stadthann ${ }^{9}$ ). A usser der alten Pfarrkirche beatae Mariae virginis, deren Asylrecht die Urkunde König Ludwig I. von 1380 gewährleistet, welcher die folgende Untersuchung gewidmet ist, stand im Ort ein Hospital sancti spiritus, zu dessen Unterhalt die Marienburger den dritten Weizenkübel gaben. Unter den Ortspfarrern müssen einige hervorragende Männer gewesen sein, da bis zur Reformation nächst der Stadt und Petersberg Marienburg am häufigsten Sitz des Burzenländer Decans war, so unter Franciscus und Nicolaus im 15., unter Henricus im 16. Jahrh. ${ }^{10}$ ). In der schweren Türkenzeit diente die Feste nicht allein den Ortsangehörigen, sondern auch der Bevölkerung der umliegenden Dörfer als sicherer Zufluchtsort. Als Thürme und Vorwerke

1) Urkunde der sieben Stühle Orig. Perg. Marienburg. 2) König Ludwig I. Orig. Perg. Kronstadt. 3) Nicolaus, Decan des Burzenlandes und Pfarrer von Kronstadt, und der Kronstiidter Rath, Orig. Pap. Marienburg. 4) Fridericus Pyberstain, , conservator ordinis sancti spiritus et procurator generalis ", Orig. Pap. Marienborg. 5) Origg. zu Marienburg. 6) Orig. Perg. Kronstadt. 7) Orig. Perg. Marienburg, in welcher Urkunde es heisst: eisdem senioribus civibus, populis et Saxonibus de dicta Fewlduar forum liberum singulis ebdomadis feria quinta in eadem Feulduar celebrandum de innata nobis pietatis clementia et gratia speciali duximus dandum et concedendum. ${ }^{8}$ ) J. Trausch, Uebersichtliche Darstellung der älteren Gemeinde-Verfassung der Stadt Kronstadt (Kronstadt 1865) 2. 9) Für den Umfang der eigenen Gemeinde blieben die Marienburger Marktbeamten bis in das 19. Jahrb. erste Instanz. L. J. Marienburg, Geographie des Grossfürstenthums Siebenbürgen II. $820 .{ }^{10}$ ) J. Trausch, Geschichte des Burzenländer Capituls (Kronstadt 1852) $70 \mathrm{f}$. 
in einem Bericht an den König als zu niedrig und nicht mehr ausreichend zu erfolgreicher Vertheidigung gegen die Türken geschildert worden waren, erlässt dieser in Jahre 1420 von Breslau aus den Befehl, die Festungswerke zu verstärken und zu diesen Arbeiten alle Leute der Umgegend heranzuziehen ${ }^{1}$ ). Doch konnten die Mauern nicht Schutz gewähren gegen Verwüstung des flachen Landes und Marienburg wie das ganze Burcenland hatten im 15. Jahrh. durch Türkeneinfälle arg zu leiden, so sehr, dass die Abgabe des dritten Weizenkübels an das Marieuburger Heiliggeist-Hospital unterblieb und im Jahre 1455 der Procurator Friedrich Pyberstain auf die fernere Entrichtung dieser Abgabe förmlich verzichtete ${ }^{2}$ ).

Nach der ältesten auf uns gekommenen Volkszählung aus dem Jahre 1510, Original, Papier, im Archiv der Stadt Hermannstadt und der sächsischen Nation, steht Marienburg unter den dreizehn deutschen Landgemeinden des Burzenlandes mit der Zahl der Hauswirthe an wweiter Stelle, indem Tartlau mit 230 Hauswirthen damals der volksreichste Ort ist, hierauf Marienburg mit 158, dann Zeiden mit 142 Hauswirthen folgt ${ }^{3}$ ). Auch dieses beweist mit, dass es ein kräftiges deutsches Gemeinwesen war, desseu Kirche vor allen übrigen jener Gegend durch ausdrückliche Verleihung des Asylrechtes ausgezeichnet worden ist.

Der Text der in Keınlek den 19. Juni 1380 ausgestellten Urkunde, soweit bisher bekannt geworden ist, nur in einer Ueberlieferung, auf ein Pergamentblatt geschrieben, erhalten, lautet:

"Nos Lodouicus dei gratia rex Vngariae, Poloniae, Dalmaciae etcetera notum facimus universis praesentes inspecturis, quod nos sicut ad profectum et augnentationem tidelium civium nostrorum de Feuldwar alias Marienburg sic non minus pro utilitate et commodo regni

1) Orig. Yap. Marienburg: universos et singulos populos et hospites circumsedentes, qui tempore neccessitatis et adversitatis refugium ad huiusmodi manitionem habere poterint. $\left.{ }^{2}\right)$ Derselle schreibt an 7. April 1455 aus Kronstadt: ... attentis intercessionibus quam plurimorum dominorum spiritualiom et temporalium ex parte dictorum oppidanorum et communitatis de Mergenburg propter devastationem 'Turcorum praedicti districtus Brassouiensis et annihilationem provinciae eiusdem, ratione cuius iidem homines praedicti oppidi de Mergenburg in multa damna irrecuperabilia incurrerunt, quod huiusmodi tertium cubulum tritici in posterum difficulter dare praevalebunt, de plenaria potestate wihi in hac parte concessa et miscricordia speciali eisdem villico et oppidanis de Mergenburg, ex quo convicinati sunt confinibus Turcorum, a quibus quotidie urgentur et plura patiuntur detrimenta, supplicationibus huiusmodi tamquam rutioni consonis consentiens eundem tertium cubulum tritici in posterum annuatim solvendum ... . relaxavi penitus et dimisi. Orig. Pap. Marienburg. 3) $\mathbf{A b}$ gedruckt von G. D. Teutsch im Vereinsarchiv N. F. X. $228 \mathrm{f}$. 
nostri annuimus et concessimus de plenitudine nostrae regiae potestatis et gratia speciali, ut ammodo et deinceps quicumque proscriptorum bannitorum profugorum homicidarum et aliorum pro factis licitis et honestis exulum ad ipsum oppidum nostrum Feuldwar nuncupatum intra dumtaxat murum, quo ecclesia parochialis de eadem est circumdata, confugerit, tutam, liberam et absolutam ut praemisimus intra ipsum murum sed non extra habeant standi, manendi et perseverandi facultatem absque omni metu timore et terrore rerum et personarum eorundem inhibentes et interdicentes omnibus et singulis sub poena capitum et ammissione omniun possessionum et bonorum suorum, ne buiusmodi profugos exules intra dictum murum commorantes et confugientes palam vel latenter, tacite vel expresse invadere vel aliqualiter eis nocere praesumant. Quoniam nos ex certa nostra scientia ambitum ipsius muri de Feuldwar asilum et refugium ipsorum exulum et bannitorum duximus praeficiendum et instituendum, prout hac eadem libertate aliae civitates nostrae et oppida frui et potiri dignoscuntur. Et haec volumus per fora et alia loca publica palam facere proclamari. Praesentes etiam sub magno nostro sigillo dari faciemus, dum nobis fuerint reportatae. Datum in Kemlek, feria tertia prosima ante festum nativitatis beati Johannis baptistae, anno domini millesimo trecentesimo octoagesimo."

Das Stück ist auf Pergament geschrieben und war in der heute vorliegenden Gestalt jedenfalls schon in der ersten Hälfte des 16. Jahrh. vorhanden, was aus einer dieser Zeit angehörigen Bemerkung auf dem Rücken des Stückes hervorgeht: privilegium Lodouici super libertate oppidi castri Mariae 1). Als der Kronstädter Arzt Johann Albrich in den Jahren 1713 und 1714 die ihm wichtig erscheinenden Urkunden des Kronstädter Stadtarchives ordnete, verzeichnete und abschrieb $^{2}$ ), fand eine Copie der Orkunde, die damals diesem Archiv angehörte, Aufnahme in das Palladium Coronense (Seite 20 desselben, Orig. Folioband in der Bibliothek des Kronstädter evangel. Gymnasiums; Seite 8 in der Abschrift des Palladiums im Stadtarchiv), und erhielt dieselbe auf dem Rücken die Bezeichnung: Lra. A. Nro. 5, dann 7 (so numerirt in Albrichs Index, geschrieben ron Lucas Colb, Indices chronologici, Folioband in der Kronstädter evangel. Gymnasialbibliothek). In dem der Originalhandschrift des Palladium Coronense angehängten Index setzt Albrich zu dem Regest der Urkunde: Copia. Nicht lange nach-

1) Nach diesem Wort steht noch ein Schriftzug wie das Zeichen für con jnit Abkürzungszeichen darüber (concessum?). 2 2) F. v. Löher, Archivalische Zeitschrift $\nabla .10 \%$ 
her erhielt dieselbe auf dem Rücken die Bezeichnung: Ex arca A. Lra. A. nro. 10 und ein ausfïhrlicheres Regest mit Hinweis auf die Copie des Palladium im Stadtarchiv. Zur Zeit der Entstehung des neuen, jetat noch in Gebrauch stehenden Urkundenverzeichnisses, welches von dem Archivar Georg M. G. ron Herrmann 1765 fertig gestellt worden ist, hatte sich die Urkunde schon auf Wanderschaft begeben und blieb lange Jahre hindurch unbeachtet, bis sie in dem von Joseph Trausch um das Jahr 1830 abgefassten Verzeichniss der Marienburger Urkunden mit anderen aufgefïhrt wird ${ }^{1}$ ). Vor mehreren Jahrzehnten ist sie in das Archiv des Burzenländer Capitels, endlich aus Privatbesitz wieder in das Stadtarchiv Kronstadts gekommen.

Der heimischen Literatur ist die Urkunde bis noch so ziemlich fremd geblieben. Von L. J. Marienburg, welcher in seiner Geographie II. 354 sagt, „es war vor Alters hier am westlichen Ende des MarktHeckens eine Kirche, welche das ius asyli in vorzüglichem Grade besass und deswegen berühmt war, die aber nicht mehr vorhanden ist", ist nicht sicher, ob er das Stück gekannt bat, und bis auf die neueste 'Keit wird desselben nirgends gedacht. Die Frage, ist das ein Original? unter welche Gruppe von Orkunden gehört dasselbe? führt nothwendig zur Erörterung des Urkundenwesens König Ludwig I. im Allgemeinen, ein Gebiet, welches bis noch nicht betreten ist. Obwol ich eine namhafte Anzahl von Originalen aus siebenbürgischen Archiven, ferner aus dem ungarischen Landesarchiv in Budapest benützt habe, und zur Beurtheilung wenigstens der inneren Merkmale der Ludwig'schen Urkunden solcher mehrere hundert zugänglich sind, bin ich, da ohne Zweifel der beiweiten grössere Theil der auf uns gekommenen Originale dieses Königs noch in Archiven ruht, keineswegs in der Lage nach einer oder der anderen Richtung hin abzuschliessen, sondern beschränke mich, was eben hier der Hauptzweck ist, darauf die nöthigen Richtpunkte zu gewinnen zur sachgemässen Würdigung der in Rede stehenden Urkunde für Marienburg.

Für Eintheilung der Ludwig'schen Urkunden ergeben sich ähnliche Gruppen wie für jene des fast während derselben Jahre regierenden deutschen Kaisers Karl IV. und desselben Nachfolger aus dem Hause Luxemburg. Bei Berücksichtigung der versehiedenen äusseren und inneren Merkmale der Urkunden scheiden sich diese in folgende Gruppen: Diplome, Mandate und Briefe ${ }^{2}$ ).

1) Indices chronologici, Folioband, Kronst. evangel. Gymnasialbibliothek. $\left.{ }^{2}\right)$ Vgl. Das Urkundenwesen Karls IV. und seiner Nachfolger (1846-1487). Von Theodor Lindner (Stuttgart 1882). Beiträge zur Diplomatik der Luxemburgischen Periode ron Theodor Lindner in r. Löher's Archival. Zeitschrift IX. $168 \mathrm{f}$. 
Die Diplome lassen schon an ihrem Aeusseren ihre Sonderart erkennen; sie sind, wie alle Urkunden Ludwig I., breiter als hoch, auf grösseres Pergament und mit Aufwand grösserer Sorgfalt geschrieben. Der Anfangsbuchstabe der Urkunde, $N$, wenn diese mit Nos, oder L, wenn sie mit dem Namen des Königs beginnt, ist besonders stark gemacht und durch Schnörkel oft in recht zierlicher Art geschmückt. Dabei kommt es nicht selten vor, dass der Anfangsbuchstabe ganz fehlt, nämlich der für ihn bestimmte, in die nächsten Zeilen oder auch mitunter tiefer hinabreichende Raum zur nachherigen Eintragung leer gelassen, diese aber unterblieben ist. Aber ausserdem wurden noch andere Initialen, namentlich im Titel des Königs, dann bei Einschaltungen älterer Urkunden die Initialen dieser und der Anfangsbuchstabe der Corroboration der Ludwig'schen Urkunde fetter und grösser gemacht oder auch durch allerlei Beiwerk verziert. Einzelne Strichelchen und Schnörkel wurden auch manchmal den mitten im Text stehenden Buchstaben zugegeben, wie z. B. in dem erwähnten Marktprivileg für Marienburg von 1379. Die Schrift ist in ein gewisses Verhältuiss zum Schreibstoff gebracht, der bei Diplomen, soweit ich bisher beobachten konnte, regelmässig Pergament ist, indem nach allen Seiten hin reichlich leerer Raum gelassen ist und dies strenge innerhalb eines bestimmten Linienschemas. Horizontale, schwarz gezogene oder leicht eingeritzte Linien dienen dem Schreiber zur Richtschnur und je zwei oder eine senkrechte Linie zu beiden Seiten der Schrift halten diese in bestimmten Grenzen, welche gewöhnlich nur auf der Seite der Zeilenenden etwas überschritten wird, wenn es sich darum handelt, noch einen oder mehr Buchstaben in dieser Zeile unterzubringen. Die Diplomen-Schreiber keunen keine Absätze, die Schrift geht fortlaufend bis zum letaten Wort der Urkunde. Auf dieses folgt ein Punkt oder mehrere mit einem Schnörkel.

Die Besiegelung der Diplome geschieht regelmässig durch Anbängen des grossen Doppel-Siegels an verschiedenfarbiger Seidenschnur, welche durch den unteren, umgebogenen Rand der Urkunde gezogen ist1). Nachdem dieses Siegel auf dem Feldzug Ludwigs in Bosnieu durch die zur Bewachung desselbeu bestellten Diener des königlichen Kanzlers Nicolaus diesem entwendet worden war, liess Ludwig ein neues Doppel-Siegel anfertigen ${ }^{2}$ ), mit welchem die von seinem Vater

1) Abbildung in Pray, Syntagma historicum de sigillis Tab. I. Fig. i. 2) $\Delta \mathrm{b}$ bildung in Pray a. a. O. Tab. II. Fig. 1. Ueber den Verlust des Siegels sagt Ludwig: . . quod ubi in regno nostro Boznae innumerabilis multitudo baereticorum et paternorum pululasset in errorem fidei orthodoxae ad extirpandumque de ipso regno nostro eosdem ex una parte nos personaliter instaurato valido exer- 
König Karl mit dessen erstem und zweitem Siegel besiegelten Diplome und alle jene Ludwigs, die mit dem in Bosnien in Verlust gerathenen ersten Siegel versehen waren, neu besiegelt werden mussten, um in foro Geltung zu erlangen. Die Gesetzgebung hat denn auch die Diplome Karls, welche mit dem ersten oder zweiten, und die Diplome König Ludwigs, welche vor 1364 ausgestellt und seither nicht bestätigt worden sind, für rechtsungiltig erklärt1). Das neue Doppel-Siegel, auf dem Revers selbst secundum genannt, stand seit dem Jahre 1364 in Gebrauch und wurde wie das erste den Diplomen angehängt. Sollte es zur Bestätigung eines vor 1364 ausgefertigten Diplomes verwendet werden, so wurde es neben dem vorhandenen Siegel links (vom Beschauer rechts) angehängt und in die betreffende Orkunde unterhalb des Textes die Bestätigungsformel: Ad perpetuam rei memoriam (siehe die unter Anm. 2 Seite 70 angeführten Drucke) mit dem Kanzlernamen und der Datirung eingetragen.

Die Diplome fangen mit dem Namen des Königs, mitunter auch mit einem diesem vorgesetzten Nos an. Invocation ist mir in Originalen Ludwigs überbaupt nur in Urkunden für Polen vorgekomunen. Die beiden Urkunden aus dem Jahre 1380 für Kaufleute aus Ungarn und Polen und für die galizische Stadt Sandec, welche Gustav Wenzel reröffentlicht hat, haben allerdings im Eingang die Invocation: In nomine domini amen; aber es sind diese Urkuuden nicht im Original, sondern in einem Copialbuch des 16. Jahrh. erhalten ${ }^{2}$ ), hingegen andere mit derselben Invocation, gleichfalls für Polen, im Original vorhanden ${ }^{3}$ ). Auf den Namen folgt die Devotionsformel dei gratia, dann der Titel, welcher zu dem grossen Titel des letzten Arpaden (Andreas III. † 14. Januar 1301) noch: princeps Sallernitanus et honoris montis sancti angeli dominus fügt und vollständig lautet: Hungariae (oft Vngariae), Dalmaciae, Croaciae, Ramae, Seruiae, Galliciae, Lodomeriae, Cumaniac (auch Comaniae) Bulgariaeque rex, princeps Sallernitanus et honoris montis sancti angeli dominus4). Die Erwerbung

citu proficiscebamur, ex alia vero parte venerabilem in Christo patrem dominum Nicolaum archiepiscopum nostrum cancellarium, penes quem utrumque par sigilli nostri authentici habebatur, et virum magnificum dominum Nicolaum palatinum cum ceteris praefatis baronibus et regni proceribus in Wzuram destinaramus, quidam familiarium ipsius domini archiepiscopi pro custodia deputati utrumque par dicti sigilli nostri pratconcepta malitia subtraxerunt. Nos itaque praecaventes ne ex huiusmodi sigilli nostri deperditione regnicolis nostris in eorum iuribus successive periculum possit imminere, sigillum novum in duobus paribus fecimus pro nobis sculpi ... Fejér Codex IX. 3. 411, 413, 414. Codex patrius I. 221.

1) Tripartitum If. Tit. 14. $\$ 19-95$. $\quad$ 2) Wenzel, Magyar diplomácziai emlékek III. 407 und 409. 3) Ebenda 425 und 459. ") Fejér Codex IX. 1. 
Polens und die Krönung Ludwigs zum König von Polen (17. November 1370) bedingte die Aufnahme auch dieses Namens in den Titel, in welchem fortan auf Hungariae Poloniae folgte ${ }^{1}$ ).

An den Titel schliesst sich die Salutatio, allgemein gehalten, omnibus Christi fidelibus praesentium notitiam habituris salutem in omnium salvatore, oder: omnibus Christi fidelibus praesentibus pariter et futuris praesentium notitiam habituris salutem in salutis largitore. Hieran reiht sich gewöhnlich eine Arenga, deren es in Ludwigs Diplomen recht mannigfaltige gibt, worauf die Narratio und Dispositio folgen ${ }^{8}$ ). Fehlt die Arenga, so wird, bevor der Lebergang zur Narratio stattfindet, mindestens der königlichen Pflichten gegenüber dern eben in dem Diplom auftretenden Petenten Erwähmung gethan.

Das Schlussformular der Diplome enthält: Corroboratio mit Ankündigung des Siegels, Datirung mit der Formel Datum per manus N. N., worauf der Kanzler oder Vicekanzler folgt, dann das Incarnationsjahr, Tagesbezeichnung nach dem römischen Kalender und das Regierungsjahr; hieran schliesst sich die Zeugenreihe, zuerst die geistlichen, dann die weltlichen Würdenträger des Landes.

Die zweite Gruppe der Urkunden Ludwigs, für welche der Name Mandat in den Urkunden selbst gegebeu ist, indem die Dispositio durch mandamus eingeleitet wird, steht hinsichtlich der äusseren Form den Diplomen nach. Die Mandate sind einfacher gehalten, von besonderen graphischen Verzierungen findet sich in ihnen keine Spur, die Besiegelung erfolgt nie durch Anhängen des Doppel-Siegels. Die Art der Besiegelung ist indessen eine verschiedene und es kommen ansserdem verschiedene Siegel in Anwendung: der Avers des ersteu grossen Siegels mit einreihiger Umschrift auf dem Rücken der Urkunde in weisses Wachs aufgedrückt ${ }^{3}$ ), seit 1364 der Avers des zweiten grossen Siegels mit doppelreihiger Umschrift in derselben Weise befestigt ${ }^{4}$ ), oder eines der drei kleinen Siegel in rothes Wachs, papierbedeckt, innen unterhalb des Textes aufgedrückt ${ }^{5}$ ). Grösserer Haltbarkeit wegen wurde durch zwei Einschnitte in das Pergament ein Pergamentstreifen durchgezogen und das Siegel, sei es aussen oder innen, auf die sich berührenden Enden dieses Streifens aufgedrückt.

460. Codex comitum Zichy III. 208. Der mons sancti angeli ist bekanntlich der Berg Garganus, auf dem ein Kastell mit einer Kapelle sancti angeli stand.

1) Codex patrius III. $\left.208 . \quad{ }^{2}\right)$ Vergleiche ausser den eben schon citirten Diplomen noch Fejér Codex IX. 1. 467, 472, 475, 478; 2. 236, 306, 476, 479 ; S. 346,$349 ; 4.121 ; 5.370,874$. Codex patrius I. 219, 226. V. 160 . 3) Pray a. a. O. Tab. I. Fig. 7. 4) Ebenda Tab. II. Fig. 1. \$) Ebenda Tab. IV. Fig. 8, 7 und 5 . 
Zu Eingangsprotocoll, Context und Schlussprotocoll gehören in Mandaten: der Name des Königs, dem zuweilen Nos vorausgeht, die Derotionsformel mit dem kleinen Titel, bisweilen eine specielle Salutation an den Empfänger der Trkunde gerichtet, dann die Publication mit dem Auftrag (mandamus) und die Datirung, enthaltend Ausstellungsort, Tagesbezeichnung nach der feria, Incarnationsjahr ${ }^{1}$ ). Vor der Datirung wird hier und dort die Corroboratio eingeschaltet, das Siegel angekündigt und allgemeine Bekanntmachung des Mandates (per fora et alia loca publica) angeordnet. Unter dem Siegel und links oben in der Urkunde, oder an einer dieser Stellen finden sich Bemerkungen über den Referenten der in der betreffenden Urkunde verhandelten Angelegeuheit, z. B. inuen unter dem heute abgefallenen Siegel einer Urkunde von 1371: relatio Petri Zudar et Georgii fratris sui; 1378, an gleicher Stelle: propria commissio domini regis, in welchem Falle der Act vom König selbst, ohne vorherige Erstattung einer relatio, ausgegangen ist; 1373, auf dem Rücken unter dem Siegel und innen links oberhalb des Textes: relatio dominorum Stephani vairodae et Emerici palatini, wobei innen oberhalb des Textes Emericus palatinus zuerst genannt wird $^{2}$ ).

Die königlichen Briefe unterscheiden sich vou den Mandaten besonders dadurch, dass dieselben regelmässig in unmittelbarem Anschluss an den Titel, welcher auch nur rex Hungariae lauten kann, kurz die specielle Adresse des Brief-Empfängers und dieselbe auf dem Rücken ausführlich enthalten; der Name des Königs kann fehlen, die Corroboratio kommt nicht vor $^{3}$ ), und den eigentlichen Inhalt des Stückes bildet kein Auftrag, sondern eine Mittheilung oder ein Ersuchen.

Welcher dieser Gruppe muss nun die Urkunde für Marienburg zugetheilt werden? Das Aeussere der Urkunde, Freihalten entsprechenden Randes nach allen Seiten hin, die Einordnung der Schrift in ein, wenn auch nicht vollständig durchgeführtes Linienschema, Eingangswie Schlussprotocoll und Context stimmen mit den äusseren und inneren Merkmalen der Mandate überein. Somit wäre das Stück ein Mandat, aber einerseits ist der Schriftcharakter ein so grundverschiedener von dem in Originalun König Ludwigs nachweisbaren, und andrerseits fehlt die Besiegelung, ron welcher keine Spur ersichtlich

f) Fejér Codex IX. 1. 206, 208, 210, 496, 658. Codex comitum Zichy II. 285,570 . III. $327,840,4+1$. IV. 217 . - Archiv der Stadt Hermannstadt und der sächsischen Nation U. I. 24, 26, 29, $80,81,87,88,89,40$. Archiv der Stadt Kronstadt Privileg Nr. c, 9. Archiv der Stadt Klausenburg Privileg J. 33, M. 11, D. 2. 2) Archiv der Stadt Hermannstadt etc. U. I. 24, 38, 30. 3) Wenzel a. a. O. III. 414,415 . 
ist, so dass wir es hier mit einer späteren Copie zu thun haben müssen. Das lässt sich aus der Schrift im Einzelnen beweisen. Buchstabe für Buchstabe zeigt etwas Gezwungenes, Gemachtes an sich; der Schreiber ist gewohnt, ganz andere Züge seine Feder machen zu lassen, als jene, mit denen er sich ersichtlich abmüht, da er die Schwierigkeiten, mit denen er zu kämpfen hat, nicht zu verbergen vermag, woraus folgt, dass dieser und jener Buchstabe in abweichenden Formen erscheint, dass zur Schreibung eines Buchstaben der Ansatz mit der Feder bald da, bald dort gemacht worden ist, was einem gleichzeitigon Kanzleibeamten nicht nachgesagt werden kann. Gleich der dritte Buchstabe in der Urkunde, Schluss-s ist verschieden gemacht: in nos setzt die Feder oben links an, geht nach rechts in einem Grundstrich herab, dann in einem Haarstrich und Bogen nach links unten, von da rechts hinauf; ebenso 3. Zeile von unten in praesentes; dagegen in alias, 2. Zeile, setzt die Feder oben rechts an, geht im Grundstrich links herab, setzt dann nochmals frisch oben an, um den Haken zu machen, welch' letzterer eine ganz besondere Gestalt erreicht in omnibus, Z. 7. Auf ganz verschiedene Weise wird ferner gemacht $h$ in parochialis, Z. 5 , und in huiusmodi, Z. $8 ; \mathrm{r}$ in universis Z. 1 , in Feuldwar Z. 2, in proclamari $Z$. 3 v. u., tercia $Z .2$ v. u.; abweichend sind auch gemacht die Abkürzungen für que in quicumque Z. 3 und absque Z. 6 , dann für per Z. 6 und Z. 3 v. u. Der allgemeine Abkürzungsstrich nimmt alle denkbaren Formen an, bald nach oben, bald nach unten gebogen, oder einfach geschweift oder eingekerbt. Dem Schreiber hat, das lässt sich schliessen aus der ganzen Anlage der Copie, das Original der Urkunde vorgelegen, wenigstens sieht man deutlich die Absicht des Schreibers, seine Arbeit einem Original-Mandat anzupassen; sogar die Punkte mit Schnörkel, welche so wie hier oder ähnlich sich in Mandaten finden, hat er hinter das letzte Wort, octoagesimo, gesetzt 1 ) Dass die Copie verhältnissmässig spät entstanden ist, dafür spricht hauptsächlich der Mangel an Vertrautheit mit der damaligen, König Ludwig gleichzeitigen Schrift, welchen der Schreiber verräth. Die Accente auf $u$, in murum Z. 6 und 8, weisen nicht weniger mindestens auf das 15. Jahrh. hin. $\mathrm{Ob}$ aber die Ueberlieferung glaubwïrdig, die Urkunde selbst echt ist, ist nunmehr festzustellen.

Name, Devotionsformel und Titel in den älteren Mandaten lauten, mit oder ohne vorausgeschicktes Nos: Lodouicus dei gratia rex Hungariae, wozu nach der Vereiniguug Polens mit Ungarn (1370) noch

1) Vgl. Archiv der Stadt Hermannstadt etc. U. I. 29, 30, 31, 87, 39. 
kommt: Poloniae, Dalmatiae etcetera ${ }^{1}$, wie denn auch die Vorlage diesen erweiterten Titel aufweist. Von Urkunden, welche für Sicilien ausgestellt sind ${ }^{2}$ ), abgesehen, sind nur selten und geringe $\mathrm{Abweichungen}$ zu verzeichnen, wie 1371: Lodouicus dei gratia rex Hungariae et Poloniae ${ }^{3}$ ), Lodouicus dei gratia rex Hungariae, Poloniae et Dalmatiae $\left.{ }^{4}\right)$. Es folgt in unserer Urkunde die Publicationsformel: notum facimus universis praesentes inspecturis, quod nos ..., gleichwie in Hermannstädter Originalen aus den Jahren 1372 und 1382; der König knüpft daran die Versicherung, dass er überhaupt auf das Wol seiner Getreuen von "Feuldwar alias Marienburg " bedacht sei ${ }^{5}$ ) und umschreibt sodann eingehend das Asylrecht der dortigen Pfarrkirche, welches Recht sie in demselben Umfang erbält, "prout hac eadem libertate aliae civitates nostrae et oppida frui et potiri dignoscuntur." Eine Proclamationsformel, die nicht nothwendiger Weise in allen Mandaten stehen muss, aber hïufig vorkommt, ist zwischen Disposition und Corroboration eingeschaltet: Et haec volumus per fora et alia loca publica palam facere proclamari; wörtlich gleichlautend findet sich diese Formel z. B. in einer Urkunde für Agnetheln aus dem Jahre 1376 ) und in einer Lrkunde von 13807); ähnlich heisst es in anderen Urkunden, als 1369: et haec volumus palam facere et in foris proclamari $\left.{ }^{8}\right) ; 1375$ : et haec in foris et aliis locis publicis dictarum partium Transsiluanarum, ubi necesse fuerit, palam iubemus et volumus facere proclamari ${ }^{9}$ ); 1377 : et haec volumus inibi palam facere proclamari ${ }^{10}$ )

Corroboration und Ankündigung des Siegels lauten: Praesentes etiam sub magno nostro sigillo dari faciemus, dum nobis fuerint reportatae, übereinstimmend mit anderen Mandaten Ludwigs, so mit einer Kronstïdter Orkunde aus dem Jahre 1374'11) und einer Urkunde von 1382 für den königlichen Schatzmeister Nicolans ${ }^{12}$ ). Nur wenig

1) Siehe ausser deu oluen S. 7:; ejwähnten Mandaten noch Originale in den Archiven zu Bistritz, Hermannstallt, Klausenburg und Kronstadt. ' ?) So 1350 für den deutschen Orden in regno Siciline: Vngariae, Jerusalem et Siciliae rex. Wenzel a. a. O. II. 887 . 3) Codex patrius V. 156. 4) Orig. in Klausenburg. - Einmal ist rex ausgelassen worlen, 1:i6, da der'litel lautet: Nos Lodouicus dei gratia Hungariae, Poloniae, Dalmaciae etcetera. Die Urkunde ist mittelbar bestätigt von König Sigmund 1421. Pesty, A szörényi bánság és ozörény vármegye története III. 6. 5) Vgl. Fejér Codex IX. F. 157 zum Jahre 1377. ๑) Reschner Diplomatarium IX. 116 oben. 7) Fejér Codex IX. 5. 395. 9) Ebenda IX. 4. 1i1. 9) Orig. im Stadtarchiv Dees. In diesem Stück folgt die Proclamationsformel als ursprünglich ausgelassen auf das Incarnationsjahr, weshalb hinter proclamari noch gesetzt wurde: Datum ut supra. 10) Fejér Codex IX. 5. 15i. 11) Eingeschaltet von König Sigmund 1:95, Orig. Kronstadt. 12) Fejér Codex IX. 5. 568. 
weicht davon ab ein Kronstädter Original von 1377, in welchem steht: quas sub magno nostro sigillo dari faciemus, dum nobis fuerint reportatae. In einem Stück von 1366 ist zu lesen: praesentes enim sub maiori nostro sigillo privilegialiter emanari faciemus, dum nobis fuerint reportatae 1 ).

In vollem Einklang mit Ludwigs Mandaten steht die Datirung der Marienburger Urkunde, da selbe lautet: Datum in Kemlek, feria tertia proxima ante festum nativitatis beati Johannis baptistae, anno domini millesimo trecentesimo octoagesimo. Regel ist, dass nach Datum der Ausstellungsort, dann die Tagesdatierung, gewöhnlich die Ferialbezeichnung mit Rücksicht auf ein kirchliches Fest oder einen Heiligentag, endlich das Incarnationsjahr als , annus domini “. folgt, z. B. Datum in Vissegrad, in festo beati Nicolai confessoris, anno domini ${ }_{M}^{m o} \operatorname{CCC}^{m \prime} \mathrm{L} \mathrm{XXmo} \mathrm{quinto}{ }^{2}$ ), oder: Datum in Hothuon, feria quinta proxima post octavas festi Pascae, anno domini M̂CCCิ L XX̃octaro ${ }^{3}$ ). Anders sind Urkunden datirt, deren Empfänger in Italien ist; solche haben Tagesbezeichnung nach der Ordnungszahl und dem Monatnamen, neben dem Incarnationsjahr auch die Indiction und das Regierungsjahr sowol "Vngariae" als auch "Jerusalem et Siciliae"4). Es bleibt noch ein Punkt der Datirung zu prüfen: stimmt der hier angegebene Aufenthaltsort Kemlek, worunter wol Kömlöd, deutsch: Kemling, bei Tolva zu verstehen ist, zu dem Itinerar?

Das Itinerar König Ludwigs für das Jahr 1380 stellt sich auf Grund der von mir benützten Urkunden in folgender Weise zusammen. Gegen Finde des Jahres 1379 befindet sich Ludwig in Neusohl, wo ich denselben noch am 25. November finde; hier hält er sich auch am 12. Februar 1380, von welchem Tage die erste mir bekannt gewordene Urkunde dieses Jahres datirt ist, in der der Ausstellungsort verzeichnet wird. König Ludwig urkundet also:

in dominica Invocavit . . . . . . Februar 12 in Neusohl ${ }^{5}$ )

feria tertia proximo post dominicam Invocavit . . . . . . . Februar 14 in Neusohl ${ }^{6}$ ) in dominica Laetare . . . . . . März 4 in Neusohl7) sabbato proximo ante dominicam Ramispalmarum - März 17 in $\left.\mathrm{Ofen}^{8}\right)$

1) Orig. im ungarischen Landesarchiv in Budapest. ") Orig. im Stadtarchiv Dees. 3) Orig. im ungarischen Landesarchiv in Budapest, wohin dasselbe 1876 mit dem siebenbürgischen Fiskalarchiv aus Hermannstadt gekommen ist. - Vgl. ferner Codex Andegavensis IV. 321. Codex comitum Zichy III. 211 unten. Codex comitum Károlyi I. 369. 4) Wenzel a. a. O. II. ss8. 5) Codex patrius II. 188. 6) Orig. im ungarischen Landesarchiv in Budapest. 7) Fejér Codex IX. 7. $244 . \quad$ 8) Ebendas. IX. 5. 373. 
septima die octavarum festi Pascae . . April 7 in Diosgyör ${ }^{1}$ ) in quindena festi Pascae . . . . . April 8 in Visegrad ${ }^{2}$ ) sabbato proximo ante festum beati Georgii martvris

April 21 in $\mathrm{Ofen}^{3}$ ) sabbato proximo post festum heati Georgii martyris

April 28 in $\mathrm{Ofen}^{4}$ )
in vigilia festi Pentecostes . . . . Mai 12 in Ofen $^{5}$ ) ipso die festi Pentecostes . . . . Mai 13 in Ofen ${ }^{6}$ ) secuudo die festi Pentecostes. . . Mai 14 in Ofen ${ }^{7}$ ) die secunda Junii . . . . . . . Juni 2 in Agram $^{8}$ ) feria tertia proxima ante festum nativitatis Johannis baptistae . . . . Juni 19 in Kemlek in festo beati Ladislai regis . . . . Juni 27 in Lepsény ${ }^{9}$ ) in vigilia festi assumptionis beatae virginis August 14 in Lipcse ${ }^{10}$ ) secundo die festi nativitatis virginis . . September 9 in 0 fen ${ }^{11}$ ) octavo die termini praenotati (scil. in octa-

vis Mychaelis archangeli) . . . October 13 in Visegrad ${ }^{12}$ ) secundo die festi omnium sanctorum . . November 2 in Visegrad ${ }^{13}$ ) in festo beati Martini confessoris . . . Nov. 11 in Visegrad ${ }^{14}$ ) vigesimo die termini praenotati (scil. in

octavis festi omnium sanctorum) . Nov. 27 in Visegrad ${ }^{15}$ ) die penultima mensis Novembris . . . Nor. 29 in Neusohl 16) in festo beati Andreae apostoli . . Nov. 30 in Neusohl ${ }^{17}$ ) in festo beati Nicolai confessoris. . . December 6 in Neusohl ${ }^{18}$ ) in crastino festi beati Thomae apostoli . Decenber 22 in Lygeth 19).

Demnach befindet sich Ludwig am 2. Juni 1380 in Agram, am 19. Juni zufolge der Marienburger Urkunde in Kemling und am 27. Juni in Lepsény, südlich von Stuhlweissenburg; die Zeit vom 2. bis 19. Juni genügte ebenso, um die Strecke Agram-Kemling im Tolnaer Comitat zurückzulegen, wie es möglich war, innerhalb des 19. und 27. Juni vou Kemlek nach Lepsény zu gelangen.

1) Wenzel a. a. O. III. $837 . \quad$ 2) F'ejér Codex IX. 5. 427. $\quad$ s) Eingeschaltet von König Wladislaus I. 144\%, Orig. in Mettersdorf. 4) Fejér Codex IX. 5. 386. 5) Codex patrius VII. 417. 6) Orig. im Capitelarchiv Karlsburg. . 7) Eingeschaltet vom Kolosmonostorer Convent $1: 80$, Orig. im Capitelarchiv Karlsburg. 8) Wenzel a. a. O. III. 340. 9) Codex patrius II. 139 (Wepsen). 10) Fejér Codex IX. 5. 391 (in Lypche Zoliensi). 11) Orig. Klausenburg. 12) Codex comitum Zichy IV. $168 . \quad{ }^{13}$ ) Fejér Codex IX. 5. 382. 14) Wenzel a. a. O. IIl. 408 und 410. 15) Codex comitum Zichy $(\nabla .174$ und 176. 16) Wenzel a. a. 0. III. 415. 17) Fejér Codex IX. 5. 395. 18) Ebendas. IX. 5. 395. 19) Ebendas. IX. 6. 416 (in castro Lygeth). In demselben Ort urkundet der König am 25. Januar 1381 (in festo conversionis beati Pauli apostoli), Fejér Codex IX. 5. 486. 
Somit ergibt sich kein Verdachtsgrund gegen die Glaubwürdigkeit der Ueberlieferung der Urkunde, deren Original heute noch nicht entdeckt ist. - Die Entstehungsursache der Copie mag nur der Wunsch der Besitzer des Originals gewesen sein, von diesem für die Marienburger Kirche so wichtigen Stück ein zweites Exemplar, wenn auch nicht in beglaubigter Ausfertigung zu besitzen; dass es üblich war. solche nicht beglaubigte Abschriften anzufertigen, ist z. B. gerade aus dem Burzenland aber auch aus Hermannstadt bekannt ${ }^{1}$ ). Es mag aber - dieser Fall scheint mir hier näher zu liegen - die Abschrift vielleicht gerade in einer Zeit entstanden sein, als in Competenzstreitigkeiten zwischen geistlichen und weltlichen Behörden auch das ius asyli seitens der letzteren beanstandet worden ist. Da wird der Pleban der Marienburger Kirche es für zweckmässig gehalten haben, die werthvolle Urkunde selbst abzuschreiben oder abschreiben zu lassen. Von der Gesetzgebung war, was im Abendland schon lange zu Recht bestand, die Immunität der Kirchen und das Asylrecht derselben anerkannt ${ }^{2}$ ), nicht überall und nicht zu allen Zeiten ohne jede Anfechtung, denn bereits dem Papst Innocenz III. wird über Verletzung des Asylrechtes in Ungarn berichtet: Cum enim sicut audivimus ecclesia dei in regno Hungariae in tanta reverentia olim consueverit haberi, ut si etiam publicus latro ad eam ob tutelam sui corporis confugisset, sub eius consisteret immunitate securus, et quamdia lateret ibidem, tamquam innocens servaretur, nunc evacuata omnino tanta per iniquitatis filios libertate, non tantum nocentes personae, verum etiam res sacrae et alise, ibidem pro securitate depositae, violenter ab ecclesiis extrahuntur. Es ergeht sodann die Aufforderung an den ungarischen Episcopat, das Asylrecht der Kirche zu wahren ${ }^{3}$ ). Dass solche Freistätten, .wohin Verbrecher oder Verfolgte flüchten und Schutz geniessen kounten, in Zeiten, wo es ausser den Kirchen und deren nächster Umgebung keinen Zufluchtsort gab und die Justiz nicht uur äusserst strenge, sondern auch öfter unmenschlich vorging, als eine Art Nothbehelf sicher am Platze waren, muss ebenso zugegeben werden, wie es klar ist, dass mit dem Asylrecht auch manche Unzukömmlichkeit \%u-

1) Vereinsarchiv NF. XIX. 99 f. 2) Vgl. Rosshirt, C'anonisches Recht (Schaffhansen 185i) 506 f. - Müller, Lexikon des Kirchenrechts (2. Auflage Würzburg 18881 I. 84 f. - Dann, Ueber den Ursprung des Asylrechts in Reyscher und Wilda, Zeitschrift für deutsches Recht III. (Leipzig 1840). - Im Decret S. Stephans lib. II. cap. 51 wird die Ausnahme festgestellt: $S i$ quis in regem aut regnum conspiraverit, refugium nullum habest ad ecclesiam. - Vgl. ferner Corpus iur. Hungarici S. Ladisl. Decr. lib. II. cap. $1 \S 1$ und 2. Decr. lib. III. cap. 4 und 5. Colom. Decr. lib. I. cap. $\left.84 . \quad{ }^{3}\right)$ Katona Historia critica IV. 528. 
sammenhing. König Ludwig sieht sich 1359 geuöthigt, um dem verbrecherischen Treiben einer Mörderbande inmitten der Stadt Pressburg ein Ende zu machen, in diesem Falle das Asylrecht der dortigen Klöster, Kirchen und Kirchhöfe aufzuheben und den Rath der Stadt zu ermächtigen, die (ebelthïter wo immer zu ergreifen und abzuurtheilen ${ }^{1}$ ). Das Asylrecht des Klosters Heiligentreuz, welches diesem von König Karl 1317 für seinen Besitz in Ungarn in ausgedehntem Masse rerliehen ${ }^{2}$ ) und 1323 neuerdings bestätigt worden war $^{3}$ ), fand Ludwigs Anerkennung nicht. Als Abt Koloman im Jahre 1367 bei dem König um die Bestätigung dieser Crkuuden nachsucht, wird dem Einschreiten willfahren mit der ausdrücklichen Ausnahme, dass die das Asylrecht betreffende Stelle, welche for Allem auf den in Pressburg gelegenen Hof des Klosters Bezug hatte, fernerhin nicht mehr Geltung behalte 4 ).

Soweit Quellen darüber bis jetzt erschlossen sind, ist im 15. Jahrb. und zu Anfang des 10. bei Verhandlungen ron Streitigkeiten zwischen der politischen Gemeinde und der Burzenlïnder Geistlichkeit auch das Asylrecht besprochen, von weltlicher Seite in jedem Falle angegriffen worden. Es handelte sich vor allen Dingen um die Gerichtsbarkeit, IIm die herüber und hinüber gestritten wurde. Im Jahre 1447 erschien Thomas Armenus, Graner Propst, im Auftrage des Erzbischofs Dionysius als Visitator der dem Graner Erzbisthum unmittelbar untergestellten Decanate Hermanustadt und Kronstadt in dieser Stadt, um Frieden zu stiften \%wischen Geistlichen und Laien durch genaue Begrenzung der weltlichen wie geistlichen Gerichtsbarkeit. Dass nicht nur Kirchen an und für sich und der Raum innerhalb der Grenzen der Friedhöfe, sondern auch die Wohnungen der Geistlichen geheiligt seien, wurde dabei in den ron ihm aufgesetzten Artikeln ausdrücklich gesagt $^{5}$ ). In jener 7eit erachtete es das Burzenländer Capitel nicht

1) Fejér Codex IX. : 49 und 6. !9. - Dass König Ludwig I. die Asyle überhaupt aufgehoben babe, wie in der Geschichte von Ungarn von J. A. Fessler. ․ Auflage von Ernst Klein, II. 20i behauptet wird, erscheint nicht erwiesen. 2) Praeterea ex affluentia gratiae regalis dictis fratribus duximns annuendum, quod nulla persona cuinscumque dignitatis status aut conditionis existat, quemlibet ad domos ipsorum seu curias aliasriue possessiones quacunque de causa spe defensionis confugientem excipere, caperc vel aliam quamcumque iniuriam inferre audeat vel praesumat, et hoc praecipue in curia ipsorum fratrum Posoniensi volumus et praecipinus firmiter observari. Fontes rerum Austriacarum 2. Abth. XVI. 49. 3) Ebendas. 9.5. 4) Fejér C'odex IX. 4. s6 f. 5) Decet igitur nt domus et ecclesiae dei non solum in corpore et infra limites cimiterii sanctificati, sed etiam clomus seu loca ac dotes ipsarum ecclesiarum pro usu et habitatione presbyterorum et clericorum deputatae . . Orig. Perg. Hermannstädter Archir. Ein zweites Original im Burzenländer Capitelarchiv in Kronstadt. 
für übertlüssig, für den eigenen Gebrauch sich durch den öffentlichen Notar Vrbanus Petri de Stynawia, Kleriker der Breslauer Diöcese unter anderen Aussprüchen des Cardinals Alphonsus auch jene über das Asylrecht copiren zu lassen, welche derselbe, von Papst Nicolans 1447 darüber befragt, als Antwort gab 1 ). 1507 treffen sich die Vertreter der Geistlichkeit und der Rath von Kronstadt wieder vor Gericht, diesmal direct vor dem in Ofen weilenden Erzbischof von Gran, Thomas, welcher in den , super certis iniuriis et differentiis earundem partium " hinausgegebenen Artikeln der Immunität der Kirchen besonders gedenkt ${ }^{2}$ ). In diese Zeit des Haders zwischen kirchlicher und weltlicher Obrigkeit, etwa in die Zeit um 1500 wird die Entstehung der auf uns gekommenen Copie der Marienburger Urkunde zu versetzen sein, die nach dem Schriftcharakter von einem der Zeit der Abfassung des Originales (1380) ferne stehenden Schreiber herrührt, andererseits, wie die Bemerkung auf dem Rücken beweist, in der ersten Hälfte des 16. Jahrh. existirt hat, und zwar noch aus folgendem Grunde. Das ius asyli war durch die Reformation in Siebenbürgen nicht nur ungeschmälert gelassen, sondern es wurde dasselbe vielmehr in der ,Reformatio ecclesiarum Saxonicarum in Transylvania" Tit. XV. $4^{3}$ ), dann im Jahre 1559 durch Beschluss der sächsischen Synode ausdrücklich garantirt: „Der frayheyt halben, asylum genandt, soll in

1) Utrum fures, latrones, stuprarii, incendiarii, traditores periuri possint tueri in ecclesia et gaudere immunitate eiusdem ecclesiae.

Dicendum est quod criminosus delinquens extra ecclesiam etiam fugiens ad ipsam gaudet immunitate ecclesiae, nisi sit publicus latro vel nocturnus populator agrorum qui dum itinera frequentata vel publicas secatas obsidit aggressiones insidiis ab ecclesia extrahi potest impunitate non praestita secundum canonicas sanctiones, quibus in his standum est.

An curia plebani gaudeat tanta immunitate sicut ecclesia, cum sit extra cimiterium constructa.

Dicendum est quod si est infra quadraginta passus maioris ecclesiae vel infra triginta minoris ecclesiae gaudet immunitate alias non.

Utrum cimiterium sit violatum, dum quibus ibi in eodem capitur vel detinetur, ut reconciliari debeat ac si esset pollutum sanguine hominis.

Dicendum est quod non, si absque ipsius personae captae sanguinis effusione capiatur seu detineatur, violatur tamen immunitas ecclesiae si cimiterium est infia quadraginta vel triginta passus ut supra est declaratum et ex hoc nisi satisfaciat iniuriae ecclesiae excommunicari potest. Die Fragen und Cardinal Alphonsus' Antworten sind datirt von 1447, des Notars Vrbanus Abschrift, Orig. Perg. Burzenlănder Capitelarchiv von 1450.

2) Reschner Diplomatarium II. 422 f. ') G. D. Teutsch, Urkundenbuch der evangel. Landeskirche A. B. in Siebenbïrgen I., 0 und 65. 
allen örtern gehalten werdeu, nach königlicher mayestaett fraythumb vnd inhalt der reformation ${ }^{1}$ ). Diese Bestimmung ist auf mehreren späteren Synoden fast gleichlautend bestätigt worden, und es brauchte demnach der Pfarrer von Marienburg nicht besorgt zu sein um den Weiterbestand des Asylrechtes seiner Kirche, welches nunmehr, auch abgesehen von König Ludwigs Urkunde, gesichert war ${ }^{2}$ ). Es währte noch hundert Jahre, bis dasselbe eingeschränkt und mehr als zwei Jahrhunderte, bis es in administrativem Wege aufgeboben wurde ${ }^{3}$ ).

1) G. D. Teutsch, Lrkundenbuch der evangel. Landeskirche A. B. in Sieben. bürgen II. 24. - Vgl. H. Wittstock, Beiträge zur Reformationsgeschichte des Nösnergaues (Wien 1858) 47. J. Trausch, Schriftstellerlexikon II. 481. ") Siehe die Handschrift Codex Adamianus $83,55,112,122,250,273$, Bibliothek der evangelischen Landeskirche A. B. zu Hermannstadt. - Vgl. Teutsch a. a. O. I. 196. 3) Das bezügliche Rescript lautet:

4625.

Maria Theresia divina favente clementia Romanorum imperatrix ridua, Germaniae, Hungariae, Bohemiaeque regina, archidux Austriae, dux Lotharingiae et Barri, magna dux Hetruriae, dux Burgundiae, Styriae, Carinthiae et Carnioliae, magna princeps Transylvaniae, comes Flandriae, Tyrolis, Goritiae et Siculorum etcetera.

Mlustres, reverendi, spectabiles, magnifici, generosi, egregii, prudentes item ac circumspecti fideles nostri sincere nobis dilecti. Tam etai gloriosae memoriae antecessores nostri pro suo in religionem et iustitiam amore designandis sacris refugii locis et personis, quae ad illa confugientes asylo gandere possint, per publica edicta prospexissent, nosque ipsae etism huius rei per successivas benignas resolutiones promulgata sanctione modum proposuissemus: comperimus tamen varia circa einsmodi edictorum et resolutionum regisrum interpretationem suborta esse dubia, nec eandem ubique in regnis et provinciis nostris hereditariis observari rationem.

Quum itaque regulatio asyli a libera dispositione nostra unice dependeat, quo una ex parte differentiae inter statum ecclesiasticum et politicum saepe hactenus enatae dirimantur abususque et excessus, quos e compluribus ad nos sumtis recursibus frequentiores irrepsisse observavimus, praecareantur et antevertantur; parte vero ex altera securitatis publicae tutela et iustitiae vindicativae administratio cum sacrarum aedium reverentia concilietur, re maturo consilio perpensa, rectam, temporumque rationi et neccessitati accommodam tam pro suprema auctoritate nostra modo provisorio ferre, atque sancire decrevimus normativam ideam tam a sacris ecclesiarum antistitibus, quam a tribunalibus et magistratibus omnibusque iure asyli gaudentibus exacte observandum in sequentibus.

Primo quod ab immunitate sacris locis a principum pietate et munificentia concessa omnino exclusi sint sequentes, si ad illa confugerint, delictorum publicorum rei videlicet: rei laesae maiestatis divinae, iniurii in sanctos, caedium in templis, vel caemeteriis commissorum auctores, fures sacrilegii, latrones et gras- 
Fasst man die Folgerungen der Untersuchung zusammen, so ergibt sich, dass die vorliegende Ueberlieferung verlässlich, die Urkunde

satores viarum, nocturni agrornm depopulatores, sicarii sive ipsi proditorio modo hominem occiderint, sive operam ad id commodarint, sive alterius opera usi sint. Rei lacsae maiestatis humanae illicitae conspirationis ac perduellionis. Adultern. tores monetae quod omnes species, imitatores dolosi sigillorum, chartarum signa. tarum et chyrographorum publicorum. Qui homines, fontes, puteos, vel pascui veneno infecerint. Incendiarii, vel qui occasione incendii eluvionis, naufragii aliarumque id genus calamitatum easdem furtis auxerint. Expilatores aerarii et peculii publici, eiusque debitores et qui commissa sibi pecuniae publicae administratione dolose in rem propriam usi fuerint. Et denique cuiuscunque generi: fures, infanticidae, procuratores abortus, etiam si non, nisi operam suam contulerint. Raptores virginum. Qui ob einsmodi crimina excepta detenti fractis carceribus effugerint, decoctores patrimonii, dolosi vectigalium defraudatores, militiae desertores, et qui legitimi magistratus iussa facientem occiderint vel vulnere affecerint.

Secundo immunitas localis deinceps nonnisi templis sacrisque aedibus com. petet, in quibus religionis christianae sacramenta administrantur, vel eucharistia asservatur; monasteris rero, collegia et aedes religiosorum vel aliorum ordinis ecclesiastici hominum, et multo magis publicas scholae, xenodochia, et pia loca alia immunitate ista non gaudebunt, nec quoad asylum alia ratione, quam civium domus habenda sunt.

Tertio. In procedendi modo haec observari benigne volumus. Si quis in sacram aedem confugerit, magistratus civilis sive index sine mora illius traditionem a templi antistite humaniter petet, qui eam statim, etiam sine praevia superioris ecclesiastici consultatione aut concessione praestabit, si minus, confuga a magistratu civili sive iudice apprehendetur et sine strepitu quantum fieri potest extrahetur.

Quarto. Facta confugae extractione idem magistratus cognoscet, an illius delictum sit generis in praesenti edicto exceptorum nec ne? si primum, contra confugam procedet, ac si nunquam in loco asyli fuisset, si alterum, restituet ipsum loco, unde fuerat extractus.

Quinto. Occultationes vel subductiones confugarum quocumque modo, rel obtentu non tententur, sub gravissima indignationis nostrae poena vetamus. Tribunalibus vero et magistratibus nostris benigne praecipimus, ut si quae persona ecclesiastica, monasterium vel religiosa familia contra istam prohibitionem sive per se, sive per alium egerit, non solum ad damni, si quod propterea illatum fuerit, resarcitionem adigantur, sed etiam mulcta pecuniaria coerceantur indispensabiliter, servatis nihilominus de iure servandis audiendi et constituendi.

Quemadmodum proinde serio volumus, ut omnes tam esclesiastici et religiosi, quam seculares cuivscumque ordinis vel conditionis praeadducta universa a nobis benigne decreta constituta et sancita exacte observent et exequantur, utque hoc ab aliis quoque fiat, sedulo curent, ita vestrum erit, ea, hunc in finem sparsis circularibus ad omnium, quorum interest, notitiam promulgare. Quibus in reliquo gratia nostra caesareo regia ac principali benigne propensae manemus. Datum in civitate nostra Wienna Austriae, die vigesima mensis Augusti, anno domini 
K. Ludwig I. Urk. v. 1380 üb. d. Asylrecht d. Marienburger Kirche.

König Ludwig I. von 1380 für die Kirche zu Marienburg echt ist und die Entstehung der Abschrift in die Zeit um 1500 fällt.

millesimo septingentesimo septuagesimo septimo, regnorum vero nostrorum trigesimo septimo.

Maria Theresia $\mathrm{mp}$.

Thadaeus baro a Reischach mp.

Ad mandatum sacrae caesareate regiaeque maiestatis proprium

Alexander Horváth mp.

Ein Druckbogen in folio, Archiv der Stadt Hermannstadt und der aächoischen Nation, Acten 17i: Nr. i:.

Vgl. Jo. Szegedi, Tripartitum iuris Hungarici tyrocinium (Tyrnaviae 1767.8.) S. 499 bis 508. Ferner U. v. Meltzl, Das alte und neue Kronstadt von G. v. Herrmann I. 208 und $: 01$. 